an equivalence relation only at one stage, namely, when cocycles are identified modulo coboundaries. Actually, we arrived at this definition of $H^{p}(X, A)$ while trying to set up the relative cohomology groups subject to the following two conditions. (i) $H^{p}(X, A)$ should be the cohomology group of a cochain complex associated with the pair $(X, A)$. (ii) The use of the process of identification should be minimized. Condition (i) is motivated by the desire to take advantage of the technical convenience of the general theory of cochain complexes, while condition (ii) is concerned with conceptual simplicity.

The OHio State University

\title{
EFFECTIVENESS AT THE ORIGIN OF THE SUM SET OF BASIC SETS OF POLYNOMIALS
}

RAGY AND BUSHRA H. MAKAR

We have recently investigated the effectiveness [2] of the sum set

$$
\begin{aligned}
\left\{u_{n}(z)\right\}=\frac{1}{\lambda+\mu+\cdots+\gamma}\left[\lambda\left\{p_{n}(z)\right\}+\mu\left\{q_{n}(z)\right\}\right. & +\cdots \\
& \left.+\gamma\left\{t_{n}(z)\right\}\right],
\end{aligned}
$$

$\lambda+\mu+\cdots+\gamma \neq 0$, in a circle in which the constituent simple monic sets $\left\{p_{n}(z)\right\},\left\{q_{n}(z)\right\}, \cdots,\left\{t_{n}(z)\right\}$ are each effective. We have also investigated the order [3] of the set $\left\{u_{n}(z)\right\}$ when the constituent sets are of a given order. It is proposed here to investigate the effectiveness at the origin of the set $\left\{u_{n}(z)\right\}$ when its constituent sets are each effective at the origin.

The set $\left\{p_{n}(z)\right\}$ is said to be effective at the origin [4] when it represents every function regular at the origin in some circle surrounding the origin. If the representations of powers of $z$ by the set $\left\{p_{n}(z)\right\}$ are given by

$$
z^{n}=\sum_{i=0}^{n-1} \pi_{n i}(p) \cdot p_{i}(z)+p_{n}(z)
$$

the necessary and sufficient condition that the set $\left\{p_{n}(z)\right\}$ is effective at the origin is that [4]

Received by the editors May 28, 1952. 


$$
\lambda(0+, p)=\lim _{R \rightarrow 0+} \lambda(R, p)=\lim _{R \rightarrow 0+}\left[\limsup _{n \rightarrow \infty}\left\{\omega_{n}(R, p)\right\}^{1 / n}\right]=0,
$$

where

$$
\begin{aligned}
& \omega_{n}(R, p)=\sum_{i=0}^{n-1}\left|\pi_{n i}(p)\right| A_{i}(R, p)+A_{n}(R, p) ; \\
& A_{i}(R, p)=\max _{|z|=R}\left|p_{i}(z)\right| .
\end{aligned}
$$

Let us consider the two simple monic sets $\left\{p_{n}(z)\right\},\left\{q_{n}(z)\right\}$ defined by

$$
\begin{aligned}
& p_{n}(z)=z^{n} \quad \text { when } n \text { is even, } \\
& p_{n}(z)=2 z^{n-1}+z^{n} \text { when } n \text { is odd, } \quad p_{0}(z)=1, \\
& q_{n}(z)=2 z^{n-1}+z^{n} \text { when } n \text { is even, } \\
& q_{n}(z)=z^{n} \quad \text { when } n \text { is odd, } \quad q_{0}(z)=1 .
\end{aligned}
$$

Each of $\left\{p_{n}(z)\right\}$ and $\left\{q_{n}(z)\right\}$ is effective at the origin. The set

$$
\left\{u_{n}(z)\right\}=\left[\left\{p_{n}(z)\right\}+\left\{q_{n}(z)\right\}\right] / 2
$$

is defined by

$$
u_{n}(z)=z^{n-1}+z^{n}, \quad n \geqq 1, \quad u_{0}(z)=1,
$$

and is not effective at the origin.

Thus in general if the constituent sets $\left\{p_{n}(z)\right\},\left\{q_{n}(z)\right\}, \cdots$, $\left\{t_{n}(z)\right\}$ are each effective at the origin, the sum set $\left\{u_{n}(z)\right\}$ in (1) is not necessarily effective at the origin.

In our recent papers we have defined an algebraic basic set $\left\{u_{n}(z)\right\}$ as the set whose matrix of coefficients $U=\left[u_{i j}\right]$ 'satisfies an algebraic equation, and have shown that an algebraic simple monic set $\left\{u_{n}(z)\right\}$ of degree $m$ is such that

$$
(U-I)^{m}=0,
$$$$
1 \leqq m<\infty .
$$

It is evident that the matrix $U$ associated with the set $\left\{u_{n}(z)\right\}$ in (2) does not satisfy such an algebraic equation. We are again led to consider algebraic sum sets $\left\{u_{n}(z)\right\}$; when we assume that the sum set $\left\{u_{n}(z)\right\}$ in (1) is algebraic, we obtain the following result.

Theorem. If $\left\{p_{n}(z)\right\},\left\{q_{n}(z)\right\}, \cdots,\left\{t_{n}(z)\right\}$ are simple monic sets of polynomials effective at the origin, and the sum set

$$
\left\{u_{n}(z)\right\}=\frac{1}{\lambda+\mu+\cdots+\gamma}\left[\lambda\left\{p_{n}(z)\right\}+\mu\left\{q_{n}(z)\right\}+\cdots+\gamma\left\{t_{n}(z)\right\}\right]
$$


$\lambda+\mu+\cdots+\gamma \neq 0$, is algebraic, then $\left\{u_{n}(z)\right\}$ is effective at the origin.

Let $\left\{u_{n}(z)\right\}$ be algebraic of degree $m$; then

$$
(U-I)^{m}=0 \text {. }
$$

Expanding, $U$ being self-associative, and multiplying by $\Pi(u)$ $=\left[\pi_{n i}(u)\right]$, the reciprocal of $U$, we obtain

$$
\Pi(u)=m_{c_{1}} I-m_{c_{2}} U+m_{c_{3}} U^{2}-\cdots+(-1)^{m-1} U^{m-1} .
$$

Writing $U^{v}=\left[u_{n d}^{(\nu)}\right]$, this gives

$$
\pi_{n i}(u)=\sum_{n=1}^{m-1}(-1)^{n} m_{c_{v+1}} u_{n i}^{(v)} \quad i \neq n .
$$

Now

$$
u_{n i}=\frac{1}{\lambda+\mu+\cdots+\gamma}\left[\lambda p_{n i}+\mu q_{n i}+\cdots+\gamma t_{n i}\right]
$$

so that

$$
\begin{aligned}
u_{n i}^{(p)}= & \left\{\frac{1}{\lambda+\mu+\cdots+\gamma}\right\}^{\prime}\left[\sum_{j, k, \cdots, l}\left(\lambda p_{n j}+\mu q_{n j}+\cdots+\gamma t_{n j}\right)\right. \\
& \left.\cdot\left(\lambda p_{j k}+\mu q_{j k}+\cdots+\gamma t_{j k}\right) \cdots\left(\lambda p_{l i}+\mu q_{l i}+\cdots+\gamma t_{l i}\right)\right],
\end{aligned}
$$

the number of factors being $\nu$.

Thus

(4) $u_{n i}^{(\nu)}=\left\{\frac{1}{\lambda+\mu+\cdots+\gamma}\right\}^{\nu}\left[\sum_{(S)} \lambda^{2} p \mu^{\circ q} \cdots \gamma^{o t}\right.$

$$
\left.\sum_{j, k, \cdots, l} \tau_{n j k} \ldots l i(S)\right]
$$

where, in $\tau_{n j k} \ldots l_{i}(S)$, the coefficients $p$ appear $s_{p}$ times, the coefficients $q, s_{q}$ times, $\cdots$, and the coefficients $t, s_{t}$ times, all permuted together according to a permutation $(S)$, and $\left(s_{p}, s_{q}, \cdots, s_{t}\right)$ is a partition of $\nu$ in positive integers and zero.

From (3) and (4) we have

$$
\begin{aligned}
\omega_{n}(R, u) \leqq \sum_{v=1}^{m-1} m_{c v+1}\left|\frac{1}{\lambda+\mu+\cdots+\gamma}\right|^{\nu}\left[\sum_{(S)}\left|\lambda^{s_{p}} \mu^{*} q \cdots \gamma^{s_{l}}\right|\right. \\
\left.\cdot\left\{\sum_{i=0}^{n-1}\left|\sum_{j, k, \cdots, l} \tau_{n j k} \cdots l i(S)\right| A_{i}(R, u)\right\}\right]+A_{n}(R, u),
\end{aligned}
$$


where $A_{i}(R, u)$ and $\omega_{n}(R, u)$ are the expressions corresponding to $A_{i}(R, p)$ and $\omega_{n}(R, p)$ associated with the set $\left\{u_{n}(z)\right\}$. It is clear that $\sum_{j, k, \cdots, l} \tau_{n j k \ldots l i}(S)=T_{n i}(S)$, say, is the coefficient of $z^{i}$ in the polynomial $T_{n}(z, S)$ where $\left\{T_{n}(z, S)\right\}$ is the product set of $s_{p}$ sets $\left\{p_{n}(z)\right\}, s_{q}$ sets $\left\{q_{n}(z)\right\}, \cdots$, and $s_{t}$ sets $\left\{t_{n}(z)\right\}$ permuted according to the permutation $(S)$. Since $\left\{T_{n}(z, S)\right\}$ is a product set of simple monic sets which are each effective at the origin, $\left\{T_{n}(z, S)\right\}$ is itself effective at the origin ([4, Theorem 38$]$ or ref. $[1$, Theorem 2]). It follows (by Theorem 17 of [4]) that

$$
\lambda(0+, T(S))=0 .
$$

Now

$$
\begin{aligned}
& \sum_{i=0}^{n-1}\left|T_{n i}(S)\right| A_{i}(R, u) \leqq \frac{1}{\lambda+\mu+\cdots+\gamma}\left|\sum_{i=0}^{n-1}\right| T_{n i}(S) \mid \\
& \cdot\left\{|\lambda| A_{i}(R, p)+|\mu| A_{i}(R, q)+\cdots+|\gamma| A_{i}(R, t)\right\} .
\end{aligned}
$$

We consider first

$$
\begin{aligned}
\sum_{i=0}^{n-1}\left|T_{n i}(S)\right| A_{i}(R, p) & \leqq \sum_{i=0}^{n-1}\left|T_{n i}(S)\right| \omega_{i}(R, p) \\
& \leqq n\left|T_{n i(n)}(S)\right| \omega_{i(n)}(R, p),
\end{aligned}
$$

where

$$
\left|T_{n i(n)}(S)\right| \omega_{i(n)}(R, p)=\max _{i}\left|T_{n i}(S)\right| \omega_{i}(R, p) .
$$

We now make use of the method of the proof of Theorem 39 of [4] (or Theorem 2 of [1]). As in the proof of that theorem:

(a) If $i(n) / n \rightarrow 0$ as $n \rightarrow \infty$, then

$$
\limsup _{n \rightarrow \infty}\left\{\sum_{i=0}^{n-1}\left|T_{n i}(S)\right| A_{i}(R, p)\right\}^{1 / n} \leqq \lambda(R, T(S)) .
$$

(b) If $\lim \sup _{n \rightarrow \infty} i(n) / n=\beta, 0<\beta \leqq 1$, then

$$
\underset{n \rightarrow \infty}{\limsup }\left\{\sum_{i=0}^{n-1}\left|T_{n i}(S)\right| A_{i}(R, p)\right\}^{1 / n} \leqq \lambda(1, T(S))\{\lambda(R, p)\}^{\beta} \text {. }
$$

Hence by (6), and Theorem 17 of [4] applied to $\left\{p_{n}(z)\right\}$, which is effective at the origin, we get

$$
\lim _{R \rightarrow 0+} \limsup _{n \rightarrow \infty}\left\{\sum_{i=0}^{n-1}\left|T_{n i}(S)\right| A_{i}(R, p)\right\}^{1 / n}=0 .
$$


Similarly,

$$
\lim _{R \rightarrow 0+} \limsup _{n \rightarrow \infty}\left\{\sum_{i=0}^{n-1}\left|T_{n i}(S)\right| A_{i}(R, q)\right\}^{1 / n}=0,
$$

and so on. Since the summations in (5) with respect to $(S)$ and $\nu$ each range over a finite number of terms, and since

$\lim _{R \rightarrow 0+} \limsup _{n \rightarrow \infty}\left\{A_{n}(R, u)\right\}^{1 / n}$

$\leqq \lim _{R \rightarrow 0+} \limsup _{n \rightarrow \infty}\left\{|\lambda| \omega_{n}(R, p)+|\mu| \omega_{n}(R, q)+\cdots+|\gamma| \omega_{n}(R, t)\right\}=0$,

we get $\lambda_{i}(0+, u)=0$, which proves the required result.

\section{REFERENCES}

1. M. T. Eweida, The effectiveness at a point of basic series and the inverse of a basic set, Proc. London Math. Soc. (1948).

2. Ragy and Bushra H. Makar, Sur la base somme de bases de polynomes, Bull. Sci. Math. (2) vol. 74 (1950).

3. - On algebraic simple monic sets of polynomials, Proc. Amer. Math. Soc. vol. 2 (1951).

4. J. M. Whittaker and C. Gattegno, Sur les séries de base de polynomes quelconques, Paris, 1949.

Cairo University 